\title{
Competing processes during the production of metal nanoparticles by pulsed laser deposition
}

\author{
J. Gonzalo,* A. Perea, D. Babonneau, ${ }^{\dagger}$ and C. N. Afonso \\ Laser Processing Group, Instituto de Óptica, CSIC, Serrano 121, 28006 Madrid, Spain
}

N. Beer, J.-P. Barnes, and A. K. Petford-Long

Department of Materials, University of Oxford, Parks Road, Oxford OX1 3PH, United Kingdom

D. E. Hole and P. D. Townsend

Science and Technology, University of Sussex, Brighton BN1 9QH, United Kingdom

(Received 14 May 2004; revised manuscript received 21 October 2004; published 24 March 2005)

\begin{abstract}
In this work we report on the competition between growth, implantation, and sputtering effects during the production of metal nanoparticles (NPs) by pulsed laser deposition. The production sequence involves first the deposition of an amorphous $(a-) \mathrm{Al}_{2} \mathrm{O}_{3}$ layer onto which gold NPs are produced. They are subsequently covered by $a-\mathrm{Al}_{2} \mathrm{O}_{3}$ and this sequence is repeated five times. The resulting nanocomposite films have metal contents per layer in a broad range: $1-9 \times 10^{15} \mathrm{at} \mathrm{cm}^{-2}$. The results clearly show the formation of two NP layers per layer of gold deposited, the deepest one consisting of NPs produced by metal implanted into the $a-\mathrm{Al}_{2} \mathrm{O}_{3}$ layer, which is acting as a substrate, and the other one consisting of NPs grown on the $a$ - $\mathrm{Al}_{2} \mathrm{O}_{3}$ surface. The high kinetic energy of a significant fraction of the Au species present in the plasma and the high fluxes $\left(10^{16}-10^{18}\right.$ at $\left.^{-2} \mathrm{~s}^{-1}\right)$ at the substrate plays an essential role in the nucleation and growth of the NPs. The competition between surface growth and sputtering at high fluence induces a self-regulation of the NP dimensions that narrows the size distributions.
\end{abstract}

DOI: 10.1103/PhysRevB.71.125420

\section{INTRODUCTION}

The development of nanoparticle (NP) synthesis and characterization techniques that make possible the control of the NPs features within a few $\mathrm{nm}$ have attracted renewed interest to the production of metal NPs, as this opens the possibility of taking advantage of their special properties for the development of applications such as new catalysts, tunnel resonance resistors, or optical devices. For many of these applications, the NPs have to be embedded in a dielectric host to form a nanocomposite.

The control of the distribution, size and size dispersion of the NPs has been a challenge for many years. Common methods to produce NPs with controlled features are energetic ion implantation ${ }^{1-3}$ or thin film technologies. ${ }^{4-6}$ Whereas the former introduces the metal into a bulk dielectric, the latter produces both the metal NPs and the host using the same deposition technique. Although sputter deposition has been the most widely used technique, ${ }^{5}$ pulsed laser deposition (PLD) has recently appeared as a promising technique due to its superior ability in producing high quality dielectric oxides. ${ }^{7}$ An alternate PLD technique using independent targets for the metal and the host has successfully been used to produce metal NPs layers embedded in amorphous $(a-) \mathrm{Al}_{2} \mathrm{O}_{3}$ with an excellent control over the in-depth separation of the NPs. ${ }^{8}$ The dimensions of the NPs are typically controlled through the number of pulses on the metal target leading to quasispherical small NPs (diameters of $2-3 \mathrm{~nm}$ ), with a narrow size dispersion (around $1 \mathrm{~nm}$ ) for low metal contents. The size and shape dispersion are found to increase as the metal content increased, and the NPs become larger and elongated as a result of coalescence and coarsening. ${ }^{9}$

The role of laser fluence used to ablate the metal target on the growth of metal NPs has been much less studied. Recent
PACS number(s): 61.46.+w, 81.15.Fg, 81.07.-b

results reported on the growth of Bi NPs using fluences in the range $0.4-5 \mathrm{~J} \mathrm{~cm}^{-2}$ show that implantation of the metal in the host is an important issue that scales with the laser fluence. For low metal contents, surface nucleation of NPs was even unexpectedly prevented at high laser fluences. ${ }^{10}$ This implantation process has been related to the high kinetic energy $(\sim 200 \mathrm{eV})$ of a significant fraction of the species involved in the PLD process. In addition to implantation, it is very well known that high kinetic energy species bombarding a surface produce sputtering. ${ }^{11}$ Such sputtering processes have been reported to occur during PLD of metals with yields as high as 0.5 and 1.0 for the deposition of Ag (Ref. 12) or $\mathrm{Zn} .{ }^{13}$ They have also been considered responsible for surface compositional changes due to preferential sputtering of the different metal components. ${ }^{14}$ Although these two processes, namely implantation and sputtering, are separately reported when producing films or NPs by PLD, they must inevitably occur simultaneously, especially when using high fluences.

The understanding of the competition between growth, implantation, and sputtering in the PLD process is thus essential to eventually achieve a fine control of the NP morphology and distribution. The aim of this work is to identify the parameters that control the role of implantation and sputtering effects on the growth of metal NPs and their incorporation in $a-\mathrm{Al}_{2} \mathrm{O}_{3}$ by PLD. The amount of deposited metal, the dimensions of the NPs and the kinetic energy of the metal species involved in the process are studied as a function of the laser fluence used to ablate the metal and $\mathrm{Al}_{2} \mathrm{O}_{3}$ targets. We have selected gold for this study because the range of kinetic energies accessible is broader than for lighter metals and because the production of Au NPs has widely been reported. To compare to earlier reports on a 
metal with lower mass such as $\mathrm{Ag},{ }^{15}$ we have also determined the velocity of $\mathrm{Ag}$ species.

\section{EXPERIMENT}

Nanocomposite films containing layers of Au NPs embedded in $a-\mathrm{Al}_{2} \mathrm{O}_{3}$ were produced by PLD in vacuum $\left(10^{-6}\right.$ mbar). An ArF excimer laser beam $[\lambda=193 \mathrm{~nm}, \tau$ $=20 \mathrm{~ns}$ full width at half maximum (FWHM)] operating at $10 \mathrm{~Hz}$ was alternatively focused on the surface of high purity $\mathrm{Al}_{2} \mathrm{O}_{3}$ and $\mathrm{Au}$ targets at an angle of incidence of $45^{\circ}$. The targets were mounted into a computer-controlled holder that allows both the continuous rotation and the sequential ablation of the targets. The growth was performed on $\mathrm{Si}$ and carbon-coated substrates held at room temperature and placed $32 \mathrm{~mm}$ away from the target surface.

The deposition rates of both the $a-\mathrm{Al}_{2} \mathrm{O}_{3}$ and the metal were independently determined in vacuum prior to the production of the nanocomposite films by means of in situ reflectivity measurements. The synthesis sequence consisted of the deposition of an $a-\mathrm{Al}_{2} \mathrm{O}_{3}$ layer to allow the NPs always to nucleate on the same type of surface. Once the NPs were produced, the matrix target was ablated to first fill in the space around the NPs and second to produce an $a-\mathrm{Al}_{2} \mathrm{O}_{3}$ layer with a certain thickness and a flat surface. ${ }^{8}$ This sequence was repeated the desired number of NPs layers and an $a-\mathrm{Al}_{2} \mathrm{O}_{3}$ protective layer was always deposited to encase the films. Further details of the synthesis procedure of these nanocomposite films can be found elsewhere. ${ }^{15}$

The dependence of the NP's morphology on the amount of metal and the laser fluence has been studied. The former has been varied through the number of pulses on the metal target, in a range from the amount of metal required to form NPs that could be imaged by electron microscopy up to the percolation threshold. The laser fluence varied from $F_{0}$ $=2.7 \mathrm{~J} \mathrm{~cm}^{-2}$ to $3.3 F_{0}=8.9 \mathrm{~J} \mathrm{~cm}^{-2}$ by changing the laser pulse energy while keeping the spot size at the target surface close to $1 \mathrm{~mm}^{2}$. The spot size was determined by placing a laser beam profiler at the position of the target surface to measure the intensity profile of the attenuated laser beam. The spot size corresponds to the area at which the laser intensity is above $10 \%$ of its maximum value. The laser fluence used to produce the matrix was in all cases the same as the one used to produce the NPs. When changing the fluence, the number of pulses on the matrix target was adjusted in order to achieve a similar spacing of the NP layers and film thickness.

Rutherford backscattering spectrometry (RBS) was used to determine the metal content of the films deposited on $\mathrm{Si}$ substrates, the film thickness and the in-depth separation between NP layers. ${ }^{8}$ A $2.0 \mathrm{MeV}{ }^{4} \mathrm{He}^{+}$beam was used and the experimental spectra were analyzed using the RUMP code, the error in the determination of the gold content being $2 \%$.

The analysis of the morphology and the crystallographic structure of the NPs was carried out using high resolution electron microscopy (HREM) and selected area electron diffraction (SAD) using a JEOL 4000EX TEM operated at $400 \mathrm{kV}$ with a point-to-point resolution of $0.16 \mathrm{~nm}$. The outof-plane morphology and distribution of the NPs was studied

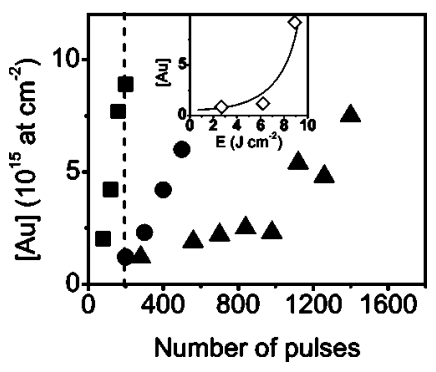

FIG. 1. Areal density of $\mathrm{Au}$ atoms per NP layer $([\mathrm{Au}])$ as a function of the number of laser pulses used to produce each NP layer, for increasing laser fluences: $(\mathbf{\Delta}) F_{0} ;(\boldsymbol{O}) 2.3 F_{0}$; and (ם) $3.3 F_{0}$, where $F_{0}=2.7 \mathrm{~J} \mathrm{~cm}^{-2}$. The inset shows the evolution of $[\mathrm{Au}]$ as a function of the laser fluence for layers grown using 200 laser pulses.

in cross-section specimens having 5 NP layers produced on $\mathrm{Si}$ substrates. They were prepared using a focused $\mathrm{Ga}$ ion beam (FIB) milling system. The in-plane morphology of the NPs was studied in samples grown on carbon-coated mica substrates with a simple sandwich structure of $\mathrm{Al}_{2} \mathrm{O}_{3} / \mathrm{NPs} / \mathrm{Al}_{2} \mathrm{O}_{3}$ to prevent the overlapping of images from different NP layers. The films were floated off the carbon-coated mica substrate in de-ionized water and picked up on copper grids for observation, having a typical size of $1 \times 1 \mathrm{~mm}^{2}$. Within this area, the structural features of the specimens were homogeneous. The analysis of the images was performed by manually outlining the NPs to produce binary images that were processed using the Gatan Digital Micrograph software to determine the size, shape and number density of the NPs. The estimated error in the determination of the NPs size and number density was estimated to be $10 \%$.

Finally, the velocity of $\mathrm{Au}, \mathrm{Ag}$, and $\mathrm{Al}$ excited atoms in the plasma was measured by spatially resolved real-time optical emission spectroscopy using the method described elsewhere. ${ }^{16}$ The plasma produced by laser ablation was imaged at the entrance slit of a spectrometer and scanned along the normal to the target. The analysis of the emission transients recorded for different distances to the target allowed us to determine the most probable velocity. The velocities were determined within $10 \%$ by averaging the results for the $481.2 \mathrm{~nm}$ and $627.8 \mathrm{~nm}$ lines in the case of $\mathrm{Au}$, the $466.8 \mathrm{~nm}, 447.6 \mathrm{~nm}$ and $546.6 \mathrm{~nm}$ lines in the case of $\mathrm{Ag}$ and the $394.4 \mathrm{~nm}$ line in the case of Al. All of them correspond to neutrals, since no emission lines related to $\mathrm{Au}^{+}$ could be detected in the present experiments.

\section{RESULTS}

Figure 1 shows the dependence of the areal density of gold atoms per layer $\left([\mathrm{Au}]\right.$, in at. $\left.\mathrm{cm}^{-2}\right)$ on the number of pulses on the Au target. The results obtained for the three laser fluences studied are all included. The $[\mathrm{Au}]$ content increases as the number of laser pulses increases, with the increase becoming faster as the fluence increases. For the case of the films produced at the lowest fluence $F_{0}$, the data clearly show a change of slope for $[\mathrm{Au}]$ values close to 


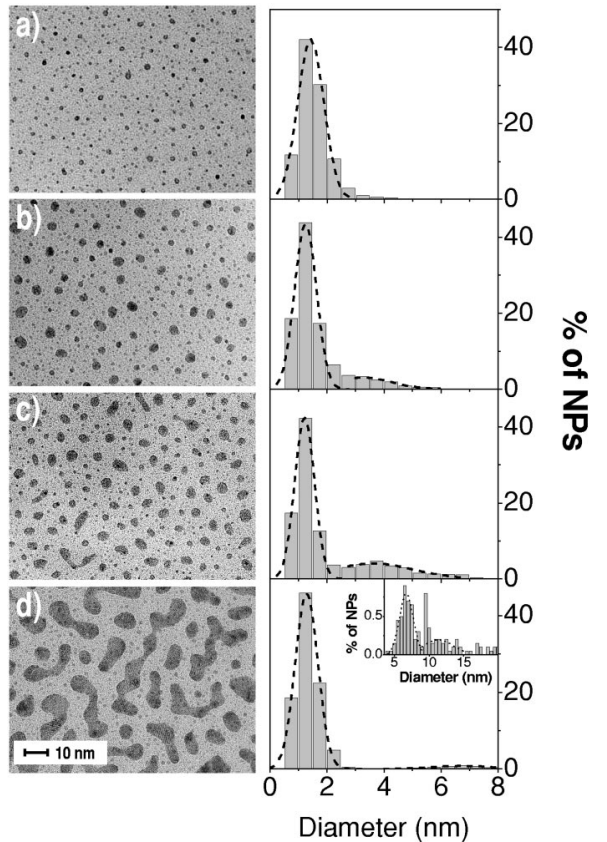

FIG. 2. Plan-view HREM images (left) and dispersion in the NP diameter (right) for films containing $\mathrm{Au}$ NPs produced using the highest laser fluence $\left(3.3 F_{0}\right)$ and having increasing $[\mathrm{Au}]$ values $\times 10^{15}$ at. $\mathrm{cm}^{-2}$ of (a) 2.0, (b) 4.2, (c) 7.7, and (d) 8.9. The inset in (d) (right) shows the same dispersion but for NP diameters greater than $5 \mathrm{~nm}$ on a magnified scale.

$2.1-2.5 \times 10^{15}$ at. $\mathrm{cm}^{-2}$. The inset shows $[\mathrm{Au}]$ as a function of the laser fluence for 200 laser pulses for the three studied fluences. It is clearly seen that the amount of metal does not follow a linear trend.

Figure 2 shows plan view HREM images of films produced using the highest laser fluence $\left(3.3 F_{0}\right)$ that have increasing $[\mathrm{Au}]$ contents. For the lowest $[\mathrm{Au}][\mathrm{Fig} .2(\mathrm{a})]$, the NPs are small and approximately round. As the $[\mathrm{Au}]$ content increases, both small and large NPs are observed [Figs. 2(b) and 2(c)]. For the highest [Au] [Fig. 2(d)], a network of elongated metal features close to the percolation limit is observed in addition to the small NPs. Since some NPs become elongated due to coalescence, the in-plane dimensions of the NPs have been determined from the HREM images by defining for each NP a length (the longer dimension) and a breadth (the in-plane dimension in the direction perpendicular to the length). Figure 3 shows the in-plane aspect ratio of both the large and small NP, defined by their length to breadth ratio as a function of $[\mathrm{Au}]$. Note that these characteristic dimensions are not well defined for the film produced at the highest fluence with the largest [Au] content [Fig. 2(d)], and thus no point is plotted for the large NPs in this case. It is clearly seen that independently of the laser fluence used, the aspect ratio is approximately constant and equal to 1.3. For the statistical purposes considered in the present work we have characterized the NPs with an equivalent diameter calculated as the mean value of the length and the breadth. The dispersion of the diameter of the NPs produced at the highest fluence as a function of $[\mathrm{Au}]$ is shown on the right-hand side of the corresponding image in Fig. 2. In all cases, a narrow distribution centered at $1.4 \pm 0.2 \mathrm{~nm}$ is ob-

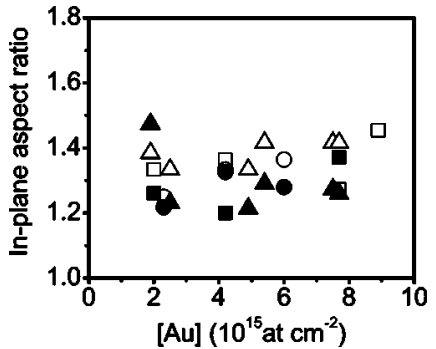

FIG. 3. In-plane aspect ratio of $\mathrm{Au}$ NPs as a function of $[\mathrm{Au}]$ for NPs produced using laser fluences of $(\triangle, \mathbf{\Delta}) F_{0} ;(\bigcirc, \bullet) 2.3 F_{0}$; and $(\square, \square) 3.3 F_{0}$. The open and filled symbols, respectively, correspond to small and large NPs.

served. Whereas the film having the lowest $[\mathrm{Au}]$ content only exhibits this narrow distribution, an additional broader distribution appears when increasing the $[\mathrm{Au}]$ content, the maximum of this second distribution shifting to longer diameters as $[\mathrm{Au}]$ increases. This bimodal distribution for the film having the highest $[\mathrm{Au}]$ content is better appreciated in the inset of Fig. 2(d) where the number of particles is plotted within an expanded scale.

Figure 4 shows both cross-section (left) and plan view (right) HREM images of the films with increasing [Au], having Au NPs produced using the lowest laser fluence $\left(F_{0}\right)$. The plan view images show indeed the presence of both small and large NPs in the films having higher [Au] [Figs. 4(c) and $4(d)]$. The cross section images show that the NPs are organized in layers, whose spacing is very similar (within 15\%) in all films as intended. Large and small NPs are located in
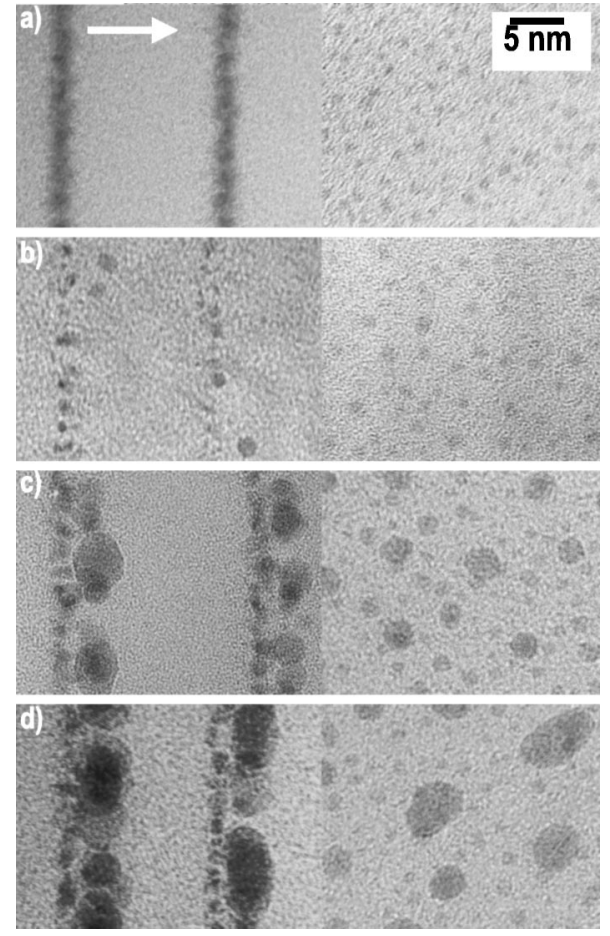

FIG. 4. Cross-section (left) and plan-view (right) HREM images of Au NPs produced using the lowest laser fluence $\left(F_{0}\right)$ and having increasing $[\mathrm{Au}]$ values $\times 10^{15}$ at. $\mathrm{cm}^{-2}$ of (a) 1.2 , (b) 1.9 , (c) 4.9 , and (d) 7.5. The arrow in (a) (left) shows the growth direction. 


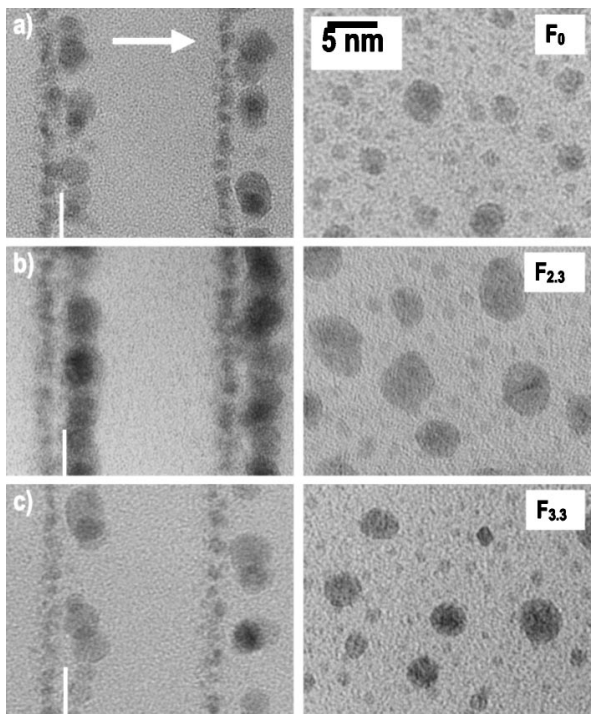

FIG. 5. Cross-section (left) and plan-view (right) HREM images of $\mathrm{Au}$ NPs having similar [Au] values and produced at increasing laser fluence: (a) $F_{0}$ and $[\mathrm{Au}]=4.9 \times 10^{15}$ at. $\mathrm{cm}^{-2}$; (b) $2.3 F_{0}$ and $[\mathrm{Au}]$ content $=4.2 \times 10^{15}$ at. $\mathrm{cm}^{-2} ; \quad$ (c) $3.3 F_{0}$ and $[\mathrm{Au}]=4.2$ $\times 10^{15}$ at. $\mathrm{cm}^{-2}$. The arrow in (a) (left) shows the growth direction. The vertical lines in (a), (b), (c) (left) mark the approximate location of the deposition surface prior to the NP deposition cycle.

separate layers, with the small NPs lying beneath the large ones. The film having the smallest [Au] [Fig. 4(a)] only show one NP layer that is most likely equivalent to the deeper NP layers in the films having higher $[\mathrm{Au}]$, since only NPs with diameters of $1.4 \pm 0.2 \mathrm{~nm}$ are seen in the corresponding planview image. In the film having $[\mathrm{Au}]=1.9 \times 10^{15}$ at. $\mathrm{cm}^{-2}$ [Fig. 4(b)], a few large NPs can be seen and thus this quantity of metal appears to be close to the threshold for the formation of large NPs.

Figure 5 shows cross-section (left) and plan view (right) HREM images of films having similar $[\mathrm{Au}]$ but produced at increasing fluence (from top to bottom). In all cases, two layers corresponding to small and large NPs are observed. As the laser fluence increases, the center-to-center separation between the small and large NP layers slightly increases (from $3.0 \mathrm{~nm}$ to $4.0 \mathrm{~nm}$ ). In addition the large NPs become smaller for the highest fluence. These features are more clearly seen in Fig. 6 where the dependence of the diameter of both large and small NPs on the $[\mathrm{Au}]$ content is shown for films grown at different fluences. The diameter of the small NPs remains approximately constant independent of the laser fluence used or the $[\mathrm{Au}]$ content. The diameter of the large NPs increases as $[\mathrm{Au}]$ increases with a tendency to saturate for high $[\mathrm{Au}]$ values. Large NPs disappear for $[\mathrm{Au}]$ values $<2$ $\times 10^{15}$ at. $\mathrm{cm}^{-2}$, whereas the small ones are still seen [Fig. 4(a)]. Finally, the dependence of the diameter of large NPs on the fluence can easily be extracted from this figure following the two marked vertical lines. For low $[\mathrm{Au}]$ contents, all the laser fluences lead to the same diameter, whereas the diameter of large NPs undergoes a maximum for the intermediate fluence $2.3 F_{0}$.

The use of increased fluence to ablate the targets influences the quantity of species ablated per pulse. Figure 7

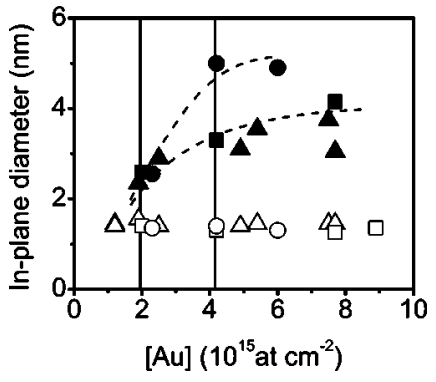

FIG. 6. In-plane diameter of $(\triangle, \bigcirc, \square)$ small and $(\boldsymbol{\Lambda}, \boldsymbol{\bullet}, \mathbf{\square})$ large $\mathrm{Au}$ NPs as a function of $[\mathrm{Au}]$ for NPs produced at increasing laser fluence: $(\triangle, \mathbf{\Delta}) F_{0},(\bigcirc, \boldsymbol{\bullet}) 2.3 F_{0}$, and $(\square, \boldsymbol{\square}) 3.3 F_{0}$. The vertical lines are guidelines to follow the dependence of the in-plane diameter at $[\mathrm{Au}]=2 \times 10^{15}$ at. $\mathrm{cm}^{-2}$ and $4.2 \times 10^{15}$ at. $\mathrm{cm}^{-2}$ as a function of laser fluence.

shows the number of atoms deposited per pulse of both the host and the metal as a function of the $[\mathrm{Au}]$ content in the deposited films. The results show that whereas the number of host atoms deposited per pulse remains constant independently of $[\mathrm{Au}]$, the number of $\mathrm{Au}$ atoms deposited per pulse unexpectedly increases as $[\mathrm{Au}]$ increases, the higher the fluence the higher the number of atoms deposited per pulse. It is worth pointing out that the number of atoms of the host deposited per pulse is approximately one order of magnitude higher than that of the metal, particularly for the lowest [Au] values.

The kinetic energy of the excited neutrals present in the plasma is deduced from real time spatially resolved optical emission transients measured in the fluence conditions used to prepare the films. For a given emission line, the time at which the maxima of the emission transients collected for a certain emission line occur, is plotted as a function of the distance to the target at which they were collected (not shown). The data follow a linear evolution whose slope provides the velocity of the corresponding species. This linear evolution is consistent with the excited atoms having a constant velocity as expected since the plasma expansion and subsequent deposition take place in a vacuum. ${ }^{16}$ The velocities experimentally determined are summarized in Table I, together with the kinetic energy calculated using these velocities. It is clearly seen that the velocity of $\mathrm{Au}$ species increases slightly with fluence, whereas that of $\mathrm{Al}$ remains approximately constant. The velocity of the $\mathrm{Ag}$ species is close to half than that of $\mathrm{Au}$.

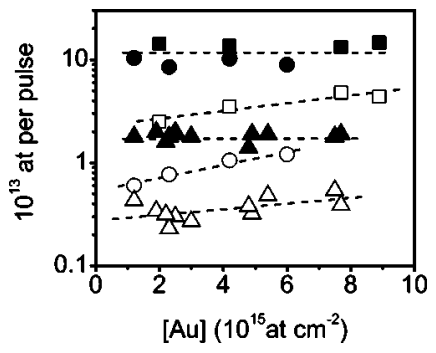

FIG. 7. Number of Au (open symbols) and host (solid symbols) atoms deposited per pulse as a function of $[\mathrm{Au}]$ in the films. The data correspond to films grown at $(\triangle, \mathbf{\Lambda}) F_{0} ;(\bigcirc, \boldsymbol{\bullet}) 2.3 F_{0}$; and $(\square, \square) 3.3 F_{0}$. The lines are linear fits to the experimental data. 
TABLE I. Velocity of Au, Ag and Al species produced by laser ablation of metal targets at different fluences, determined by optical emission spectroscopy of the plasma. $F_{0}=2.7 \mathrm{~J} \mathrm{~cm}^{-2}$ is the lowest fluence used for film growth. The kinetic energy of the species calculated from these velocities is also included.

\begin{tabular}{cccc}
\hline \hline Metal & $\begin{array}{c}\text { Ablation } \\
\text { fluence }\end{array}$ & $\begin{array}{c}\text { Velocity } \\
\left(\times 10^{3} \mathrm{~m} \mathrm{~s}^{-1}\right)\end{array}$ & $\begin{array}{c}\text { Kinetic energy } \\
(\mathrm{eV})\end{array}$ \\
\hline $\mathrm{Au}$ & $1.2 F_{0}$ & 8.3 & 70 \\
$\mathrm{Au}$ & $2.2 F_{0}$ & 9.3 & 90 \\
$\mathrm{Ag}$ & $1.7 F_{0}$ & 5.6 & 18 \\
$\mathrm{Al}$ & $1.0 F_{0}$ & 13 & 26 \\
$\mathrm{Al}$ & $2.2 F_{0}$ & 13.6 & 26 \\
$\mathrm{Al}$ & $3.7 F_{0}$ & 13.6 & 26 \\
\hline \hline
\end{tabular}

\section{DISCUSSION}

The presence of two layers of Au NPs shown by the cross-section HREM images in Figs. 4 and 5 is consistent with the bimodal distribution seen in the plan view images. This clearly suggests that different processes are taking place during the production of Au NPs by PLD. The fact that the small NPs are located deeper than the large ones suggests that they are embedded in the previously deposited $a$ $-\mathrm{Al}_{2} \mathrm{O}_{3}$ rather than being formed on its surface. These small NPs thus relate to metal incorporated in the matrix during the deposition process and we will refer to them from now on as the embedded layer, whereas the second layer of NPs is most likely formed by NPs that have been nucleated at the surface of the $a-\mathrm{Al}_{2} \mathrm{O}_{3}$ and will be referred to from now on as the surface layer of NPs.

The separate production of the two layers of NPs could only be observed for the lowest laser fluence used, since in this case, the arrival rate of the Au species is much lower. As seen in Fig. 1 for the fluence $F_{0}$, there is a change in slope in the graph of the amount of $\mathrm{Au}$ incorporated in the films as a function of the number of laser pulses. This means that for a broad range of number of laser pulses (560-980), [Au ] remains approximately constant within $2.1 \pm 0.2$ $\times 10^{15}$ at $\mathrm{cm}^{-2}$. Figure 4(b) shows that this [Au] value is the threshold for the formation of NPs at the surface since for smaller values [Fig. 4(a)], only the embedded layer is seen, while for larger ones [Fig. 4(c)], both surface and embedded layers are produced. Furthermore, this $[\mathrm{Au}]$ threshold is independent of the laser fluence used, as seen in Fig. 6.

The embedded NP layer is always observed to be present and consists of round NPs with a diameter of $1.4 \pm 0.2 \mathrm{~nm}$ independently of the $[\mathrm{Au}]$ content or the laser fluence. This result suggests that the process responsible for its formation requires a minimum $[\mathrm{Au}]$ content to initiate nucleation, but subsequent saturation is very fast. The cross-section images in Fig. 5 show that the depth position of these embedded layers with respect to the surface ones increases as the fluence is increased. We define the depth at which the embedded layer is produced, $\boldsymbol{d}$, as the distance between the center of the embedded layer and the average position of the lower surface of the NPs belonging to the surface layer, which is estimated to be the position of the $a-\mathrm{Al}_{2} \mathrm{O}_{3}$ surface, marked in Fig. 5, left by a vertical white line. The value of $\boldsymbol{d}$ increases from $1.2 \mathrm{~nm}$ to $2.0 \mathrm{~nm}$ when the laser fluence is increased from $F_{0}$ to $3.3 F_{0}$. If this embedded layer is produced by implantation of the metal in the matrix, $\boldsymbol{d}$ should depend on the kinetic energy of the Au species arriving to the surface of the $a-\mathrm{Al}_{2} \mathrm{O}_{3}$ substrate, with higher kinetic energy leading to greater implantation depth.

In the case of laser ablation of metals well above the plasma formation threshold, the ions present in the plasma play a significant role on the film deposition process, ${ }^{17}$ the ionization fraction being dependent on the laser parameters. Recent works on UV ablation of metals in the fluence range relevant here, have evidenced a significant ionization fraction $[\mathrm{Ti}(>50 \%), \mathrm{Al}(\approx 60 \%), \mathrm{Ag}(\approx 60 \%), \mathrm{Bi}(\approx 15 \%)]$ (Refs. 12, 18, and 19) and pointed out that the ion fraction seems to depend on the melting point. Considering these results and the fact that $\mathrm{Au}$ has a melting point close to that $\mathrm{Ag}$, we have estimated that in our present conditions this fraction can lie in the range 10-50\%. Moreover, since in the case of metals, excited neutrals are most likely the result of electron-ion recombination processes ${ }^{20}$ and for fluences well above the plasma formation threshold it has been reported that excited and ground neutrals have similar peak velocities, ${ }^{21}$ the velocities and kinetic energies shown in Table I can be considered a good approximation for those of the majority of the species present in the plasma.

Assuming that the variation of kinetic energy with fluence follows a linear function over the range studied, the kinetic energy of the energetic Au species would increase from $67 \mathrm{eV}$ to $115 \mathrm{eV}$ when increasing the fluence from $F_{0}$ to $3.3 F_{0}$. Using these kinetic energy values for $\mathrm{Au}$ species, a density of $2.95 \mathrm{~g} \mathrm{~cm}^{-3}$ for $a-\mathrm{Al}_{2} \mathrm{O}_{3}$ (Ref. 22), and the SRIM software, the calculated implantation ranges are $1.4 \pm 0.1 \mathrm{~nm}$ and $1.6 \pm 0.2 \mathrm{~nm}$ for $F_{0}$ and $3.3 F_{0}$, respectively. These values agree well with the experimental implantation depths determined from Fig. 5. Thus it can be concluded that the embedded layer is produced by implantation into the $a-\mathrm{Al}_{2} \mathrm{O}_{3}$ substrate of a fraction of the metal arriving at the substrate.

The existence and location of the embedded layer is also a consequence of the high Au mass. In earlier reports, an embedded layer was clearly seen when producing $\mathrm{Bi}$ NPs, ${ }^{10}$ whereas it was harder to observe in the case of Ag NPs, ${ }^{15}$ and has not been observed in the case of $\mathrm{Cu} \mathrm{NPs} .{ }^{8} \mathrm{Bi}$ has a mass very similar to that of $\mathrm{Au}$ and the velocity reported for $\mathrm{Bi}$ species in PLD processes was slightly higher (14 $\left.\times 10^{3} \mathrm{~m} \mathrm{~s}^{-1}\right){ }^{23}$ It is thus not surprising that the implanted layer is similarly well seen for both $\mathrm{Bi}$ and $\mathrm{Au}$. However, $\mathrm{Ag}$ has half the $\mathrm{Au}$ mass and the velocity of Ag species determined here is approximately half than that of $\mathrm{Au}$ species. Provided that the fluence used to produce the Ag NPs in the earlier report ${ }^{15}$ was approximately half the fluence used here to determine the velocity of excited Ag species, and assuming again a linear dependence of the kinetic energy with fluence we can estimate the kinetic energy for an energetic Ag species in Ref. 15 to be more than 8 times lower than that of an energetic Au species in this work and thus, the implantation depth for Ag had to be much smaller. This reasoning agrees well with the fact that although the existence of an implanted layer in the case of $\mathrm{Ag}$ was inferred from a dark 
contrast below the NPs in the cross-section images, a layer of embedded NPs was not formed. ${ }^{15}$ The implantation depth estimated using the velocity measured in this work is of $0.8 \mathrm{~nm}$ which is in good agreement with the experimental observations in Ref. 15. This reasoning agrees well with the fact that the metal tends to form a quasi-continuous layer rather than isolated NPs when the implantation energy is decreased. ${ }^{24}$

The kinetic energy of energetic $\mathrm{Au}$ species (close to $100 \mathrm{eV})$, and the total Au dose $\left(2 \times 10^{15}\right.$ at. $\left.\mathrm{cm}^{-2}\right)$ in the implanted layer are, respectively, at least two orders and one order of magnitude smaller than those typically used for producing NPs by energetic ion implantation. ${ }^{2,3}$ For the case of $\mathrm{Cu}$, it has been reported that there is a critical dose for forming NPs, with this threshold being lower for low ion energy. ${ }^{25}$ Although this agrees with our observations, the kinetic energies $(25 \mathrm{keV}$ and $3 \mathrm{MeV})$ for which this effect has been observed are still much higher than those involved in PLD. There is, however, another important parameter that is scarcely discussed in the literature, namely the dose rate or flux of arriving ions. This is typically in the range $10^{13}-10^{15}$ ions $\mathrm{cm}^{-2} \mathrm{~s}^{-1}$ for ion implantation. ${ }^{2,3}$ It has recently been shown that for $\mathrm{Ti}$ implantation at $9 \mathrm{keV}$ and a total dose of $6 \times 10^{16}$ ions $\mathrm{cm}^{-2}$, a decrease of the flux by a factor of two causes the metal to form a quasi-continuous layer rather than NPs. ${ }^{24}$ PLD is a transient process since most of the deposition occurs during a period of several microseconds after the laser pulse, with this deposition being repeated after a pause of some hundred milliseconds at a typical laser pulse frequency of $10 \mathrm{~Hz}$. Considering $10 \mu \mathrm{s}$ as a conservative approximation for the deposition time after each pulse, the average flux per pulse of metal species arriving at the substrate using the rates plotted in Fig. 7 is in the range of $10^{16}-10^{17}$ at. $\mathrm{cm}^{-2} \mathrm{~s}^{-1}$. These fluxes are extremely high when compared to the fluxes used during ion implantation, and thus are likely to be responsible for the formation of the embedded layer Au NPs by the implantation of energetic species. These high fluxes are also responsible for the very sharp size distribution of the embedded layer of NPs, as it has been previously observed that the size dispersion of NPs produced by ion implantation is substantially reduced when increasing the flux., ${ }^{2,3}$ Our results give thus further support to the conclusion that low energy and high flux ion implantation is required to achieve narrow NP size distributions, ${ }^{24,26}$ and provides an explanation for the narrow 2D distribution of Au NPs produced by ion implantation recently reported. ${ }^{1}$ Although the kinetic energy $(240 \mathrm{keV})$ in this report was higher than that used in the earlier ones, ${ }^{2,3}$ the flux was close to $10^{17}$ ions $\mathrm{cm}^{-2} \mathrm{~s}^{-1}$ and thus similar to the one used in this current work.

Our results clearly show that for metal concentrations per layer of $2 \times 10^{15}$ at. $\mathrm{cm}^{-2}$, the implanted portion of the metal becomes saturated and nucleation and growth at the surface starts, the diameter of the NPs increasing as the amount of metal deposited increases. The fraction of Au atoms leading to the formation of the surface layer of NPs has been estimated to increase from $\sim 50 \%$ of the total deposited Au atoms in the case shown in Fig. 2(b) up to a value close to $\sim 80 \%$ when coalescence is clearly taking place [Fig. 2(d)]. This layer corresponds most likely to the one reported earlier for PLD of $\mathrm{Cu}$ or $\mathrm{Ag} \mathrm{NPs},{ }^{8,15}$ for which the implantation effects were weak and for which the diameter of the NPs followed a nearly linear increase with the number of laser pulses in the metal target. However, similar $[\mathrm{Au}]$ values are achieved over a broad range of number of laser pulses (560980) for the lowest fluence used (Fig. 1). This [Au] content is in addition very close to the threshold for forming NPs at the surface layer as seen in Fig. 4(b). Therefore, there has to be another competing process that slows down or even prevents the growth of the NPs at the surface layers for low $[\mathrm{Au}]$ values. This conclusion is further supported by the unexpected increase of the number of Au atoms deposited per pulse as $[\mathrm{Au}]$ increases (Fig. 7) and by the sharp increase of the deposition rate at high fluence that can be deduced from the inset in Fig. 1. Since these competing processes influence the metal content but not the host content, they are most likely related to sputtering of the metal by either selfsputtering during metal deposition or by sputtering of the metal while covering the NPs with the $\mathrm{Al}_{2} \mathrm{O}_{3}$ host.

According to the classical sputtering models, the sputtering yield depends on the kinetic energy of the arriving projectiles. ${ }^{11}$ Assuming the formulation reported elsewhere based on this theory, ${ }^{14}$ and the kinetic energies in Table I, the sputtering yield of Au by host projectiles should be negligible whereas that of $\mathrm{Au}$ by $\mathrm{Au}$ projectiles would increase from 0.37 to 0.72 when increasing the fluence by a factor of 3.3. This important increase of the self-sputtering yield can account for the evolution of the NP dimensions as a function of fluence deduced from the vertical lines in Fig. 6. The fact that this evolution undergoes a maximum for the intermediate fluence is consistent with self-sputtering being dominant over growth above this fluence. This observation is consistent with the dependence of sputtering effects on fluence reported elsewhere during deposition of metals by PLD. ${ }^{12,13}$

However, the results in Fig. 7 show that the number of $\mathrm{Au}$ atoms deposited per pulse increases with the value of $[\mathrm{Au}]$ for a given fluence, even when the arrival flux of both $\mathrm{Au}$ and host species is constant. This result clearly suggests that sputtering of the metal caused by the host species must be playing a role most likely related to the special features of the PLD process that significantly differ from those of classical sputtering. ${ }^{27}$ The PLD process generates a high particle flux, and due to the low kinetic energy (compared to sputtering), they only penetrate a very short distance into the surface, and so rapidly alter the local deposit composition. Hence, the surface composition and sputtering rates must vary both with fluence and dose. Early examples, particularly for metals, showed changes in sputter yields varying by up to factors of $10 .{ }^{27,28}$ Although our results only provide clear evidence for the self-sputtering of the metal, evidence for sputtering of the metal by the host has been provided elsewhere during covering of layers of Au NPs by a $5 \mathrm{~nm}$ layer of $\mathrm{Al}_{2} \mathrm{O}_{3}$ produced by sputter deposition. ${ }^{5}$ The results show that the metal content is higher in the noncovered sample than in the covered ones only for small NPs (diameter $<2.0 \mathrm{~nm}$ ) in agreement with our own observations. We can conclude then that sputtering of the metal by host projectiles is taking place in the current work because of the very high flux during deposition of the host and is the dominant sputtering process for low $[\mathrm{Au}]$ values. 


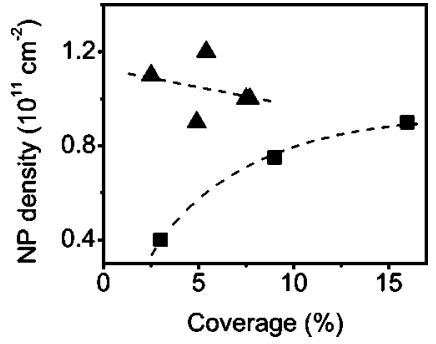

FIG. 8. Number density of surface NPs as a function of coverage for films grown at $(\boldsymbol{\Lambda}) F_{0}$ and $(\boldsymbol{\square}) 3.3 F_{0}$.

The classical theories for surface nanoparticle nucleation and growth predict that the main parameters controlling these processes are the substrate temperature and the deposition rate. ${ }^{29}$ The species arriving at the substrate diffuse on the surface until they are trapped by special sites (such as defects or nanoparticles), join other atoms to form nanoparticles or re-evaporate. There is a critical NP size below which the nuclei are unstable and thus can easily be re-evaporated or sputtered off the surface. ${ }^{4,30}$ According to Fig. 6 and Fig. 4(b), this critical size must be in our case $<2.5 \mathrm{~nm}$, or close to $2.1 \pm 0.2 \times 10^{15}$ at. $\mathrm{cm}^{-2}$. Following this reasoning, Au surface NPs smaller than this critical size are more easily sputtered off the surface than larger NPs. Once the $[\mathrm{Au}]$ content is high enough to produce NPs bigger than the critical size, these large NPs are no longer sputtered off, but remain at the surface. This preferential sputtering process of atoms and/or small NPs would also account for the reduced size dispersion observed for the Au NPs at the surface layer produced at the highest fluence, as coalescence is prevented due to sputter of the small ones. The competition between growth and sputtering processes, independent of their origin, leads to a selflimiting size effect that promotes the production of NPs with a narrow size distribution that helps to minimize coalescence.

The existence of such self-limiting effects is clearly appreciated from Fig. 8 where it is shown the evolution of the number density of NPs with the Au coverage. The number density increases for the highest fluence, while for the lowest one it is clear that it is either constant or slightly decreasing, in spite of the dispersion in the data. These results, especially the one obtained for the highest fluence, are in contrast with earlier reports for $\mathrm{Cu}$ and Ag NPs produced by PLD (Refs. 9 and 15) where the number density of NPs was found to decrease when the coverage increased, consistent with a growth process dominated by coarsening and coalescence. Similar self-limiting effects have been reported during the produc- tion of Sb NPs by cluster beam deposition, ${ }^{30}$ or transition metal NPs produced by electron-beam deposition. ${ }^{4}$ The reason why these self-limiting effects were not observed for gold in the latter work might be related to the lower flux used there and to the fact that the maximum $[\mathrm{Au}]$ content achieved was $1.38 \times 10^{15}$ at. $\mathrm{cm}^{-2}$, which is smaller than the critical value determined in this work. Self-limiting effects associated to high flux bombardment have also been reported upon high-flux $\mathrm{Cu}$ implantation in silica ${ }^{2}$ or hydrogen bombardment of carbon. ${ }^{31}$

\section{CONCLUSIONS}

Three competing processes have been identified during the growth of Au nanoparticles by pulsed laser deposition, namely the production of NPs by implantation of the metal in the host acting as substrate, nucleation and growth of NPs at the surface and sputtering of the deposited metal. The first process leads to the production of an embedded layer of very homogeneous, small NPs $(1.4 \pm 0.2 \mathrm{~nm})$ whose position, with respect to the surface scales with the kinetic energy of energetic $\mathrm{Au}$ species arriving to the substrate. This process is a consequence of the high arrival flux $\left(>10^{16}\right.$ at. $\left.\mathrm{cm}^{-2} \mathrm{~s}^{-1}\right)$ and is found to saturate in all the experimental conditions studied at a metal concentration close to $2 \times 10^{15} \mathrm{at}_{\mathrm{cm}} \mathrm{cm}^{-2}$. The second process, the nucleation and growth of NPs at the surface, starts once implantation saturates. However, sputtering of the metal at the surface by incoming Au and host species influences this process. The latter is linked to the very high fluxes and appears to dominate for low metal contents. The selfsputtering of the metal, mainly related to the increase of the kinetic energy of the species when increasing the fluence, is instead found to be dominant for high fluences. Both processes are most likely to occur simultaneously and they lead to a self-regulation of the dimensions of the NPs by preferential sputtering of the smallest NPs. This self-limiting process is more noticeable for the highest laser fluence considered and narrows the size distribution by preventing coalescence.

\section{ACKNOWLEDGMENTS}

This work was partially supported by TIC2002-03235, MCYT (Spain) and by HPRN-CT-2002-00328, EU. D.B. acknowledges financial support from the EU (Contract No. HPMF-CT-2000-00736), N.B. acknowledges support from the Somerset LEA, and J.-P.B. acknowledges financial support from the EPSRC and the EU (Contract No. HPMT-CT2000-00064).

\footnotetext{
*Electronic address: jgonzalo@io.cfmac.csic.es

'Present address: LMP, UMR 6630 du CNRS, UFR Sciences, Bâtiment SP2MI, Boulevard 3 Téléport 2, 86960 Futuroscope Cedex, France.

${ }^{1}$ K. Sun, S. Zhu, R. Fromkenecht, G. Linker, and L. M. Wang, Mater. Lett. 58, 547 (2004).
}

${ }^{2}$ N. Kishimoto, N. Umeda, Y. Takeda, C. G. Lee, and V. T. Gritsyna, Nucl. Instrum. Methods Phys. Res. B 148, 1017 (1999).

${ }^{3}$ R. H. Magruder III, R. H. Haglund, Jr., L. Yang, J. E. Wittig, and R. A. Zuhr, J. Appl. Phys. 76, 708 (1994).

${ }^{4}$ S. Gwo, C.-P. Chou, C.-L. Wu, Y.-J. Ye, S.-J. Tsai, W.-C. Lin, and 
M.-T. Lin, Phys. Rev. Lett. 90, 185506 (2003).

${ }^{5}$ J. Carrey, J.-L. Maurice, F. Petroff, and A. Vaurés, Surf. Sci. 504, 75 (2002)

${ }^{6}$ J. Carrey, J.-L. Maurice, F. Petroff, and A. Vaurés, Phys. Rev. Lett. 86, 4600 (2001).

${ }^{7}$ C. N. Afonso, in Insulating Materials for Optoelectronics: New Developments, edited by F. Agulló-López (World Scientific, Singapore, 1995), Chap. 1.

${ }^{8}$ R. Serna, C. N. Afonso, C. Ricolleau, Y. Wang, Y. Zheng, M. Gandais, and I. Vickridge, Appl. Phys. A: Mater. Sci. Process. 71, 583 (2000).

${ }^{9}$ C. N. Afonso, J. Gonzalo, R. Serna, J. C. G. de Sande, C. Ricolleau, C. Grigis, M. Gandais, D. E. Hole, and P. D. Townsend, Appl. Phys. A: Mater. Sci. Process. 69, S201 (1999).

${ }^{10}$ A. Suarez-Garcia, J-P. Barnes, R. Serna, A. K. Petford-Long, C. N. Afonso, and D. Hole, Mater. Res. Soc. Symp. Proc. 780, Y1.2.1 (2003).

${ }^{11}$ P. Sigmund, Nucl. Instrum. Methods Phys. Res. B B27, 1 (1987).

${ }^{12}$ S. Fähler, K. Sturm, and H.-U. Krebs, Appl. Phys. Lett. 75, 3766 (1999) and references therein.

${ }^{13}$ J. G. Hidalgo, R. Serna, E. Haro-Poniatowski, and C. N. Afonso, Appl. Phys. A: Mater. Sci. Process. 79, 915 (2004).

${ }^{14}$ E. van de Riet, J. C. S. Kools, and J. Dieleman, J. Appl. Phys. 73, 8290 (1993).

${ }^{15}$ J.-P. Barnes, A. K. Petford-Long, R. C. Doole, R. Serna, J. Gonzalo, A. Súarez-García, C. N. Afonso, and D. E. Hole, Nanotechnology 13, 465 (2002).

${ }^{16}$ J. Gonzalo, C. N. Afonso, and J. Perrière, J. Appl. Phys. 79, 8042 (1996); J. Gonzalo, F. Vega, and C. N. Afonso, ibid. 77, 6588 (1995).

${ }^{17}$ R. W. Dreyfus, J. Appl. Phys. 69, 1721 (1991).

${ }^{18}$ K. Sasaki, S. Matsui, H. Ito, and K. Kadota, J. Appl. Phys. 92,
6471 (2002).

${ }^{19}$ B. Thestrup, B. Toftmann, J. Schou, B. Dogget, and J. G. Lunney, Appl. Surf. Sci. 197-198, 175 (2002).

${ }^{20}$ D. B. Geohegan, in Pulsed Laser Deposition of Thin Films, edited by D. B. Chrisey and G. K. Hubler (Wiley, New York, 1994), Chap. 5.

${ }^{21}$ S. S. Chu and C. P. Grigoropoulos, J. Heat Transfer 122, 771 (2000).

${ }^{22}$ R. Serna, J. C. G. de Sande, J. M. Ballesteros, and C. N. Afonso, J. Appl. Phys. 84, 4509 (1998).

${ }^{23}$ J. Gonzalo, J. M. Ballesteros, and C. N. Afonso, Appl. Surf. Sci. 138-139, 52 (1999).

${ }^{24}$ J. P. Zhao, Z. Y. Chen, and J. W. Rabalais, J. Chem. Phys. 119, 1909 (2003).

${ }^{25}$ Y. Saito, D. Y. Shang, R. Kitsutaka, and A. Kitahara, J. Appl. Phys. 81, 3621 (1997).

${ }^{26}$ J. Olivares, J. Requejo-Isidro, R. Del Coso, R. De Nalda, J. Solis, C. N. Afonso, A. L. Stepanov, D. Hole, P. D. Townsend, and A. Naudon, J. Appl. Phys. 90, 1064 (2001).

${ }^{27}$ P. D. Townsend, J. C. Kelly, and N. E. W. Hartley, Ion Implantation, Sputtering and Their Applications (Academic, London, 1976), Chap. 6.

${ }^{28}$ P. D. Townsend, "Site characterization and aggregation of implanted atoms in materials," Proceedings NATO Summer School (Plenum, New York, 1979), pp. 339-354.

${ }^{29}$ J. A. Venables, G. D. T. Spiller, and M. Handbücken, Rep. Prog. Phys. 47, 399 (1984).

${ }^{30}$ G. Fuchs, P. Melinon, F. Santos Aires, M. Treilleux, B. Cabaud, and A. Hoareau, Phys. Rev. B 44, 3926 (1991).

${ }^{31}$ E. Salonen, K. Nordlund, J. Tarus, T. Ahlgren, and J. Keinonen, Phys. Rev. B 60, R14 005 (1999). 